\title{
Constraints on a long-range spin-dependent interaction from precision atomic physics
}

\author{
S. G. Karshenboim* \\ D. I. Mendeleev Institute for Metrology, St.Petersburg, 190005, Russia \\ and Max-Planck-Institut für Quantenoptik, Garching, 85748, Germany
}

\begin{abstract}
We present a phenomenological constraint on a pseudovector light boson beyond the Standard Model, which can induce a long-range spin-dependent interaction $\alpha^{\prime \prime}\left(\mathbf{s}_{1} \cdot \mathbf{s}_{2}\right) \mathrm{e}^{-\lambda r} / r$. In the range of masses from $4 \mathrm{keV} / c^{2}$ to those related to macroscopic distances (of $\lambda^{-1} \sim 1 \mathrm{~cm}$ ) the spin-dependent coupling constant $\alpha^{\prime \prime}$ of the electron-muon interaction is constrained at the level below a part in $10^{15}$. The constraint is weakened while extending to higher masses. The strongest constraint is related to the lepton-lepton interaction. Constraints on spin-dependent interactions of some other particles are also discussed. The results are obtained from data on the HFS interval of the ground state in muonium and a few other light hydrogen-like atoms.
\end{abstract}

PACS numbers: 36.10.Ee, 12.20.-m, 31.30.J-, 06.20.Jr, 32.10.Fn

\section{INTRODUCTION}

Muonium is a bound system, consisting of an electron and an antimuon. Interaction between the constituents is dominated by the Coulomb interaction. The major contribution to the hyperfine splitting (HFS) also comes from one-photon exchange, but from its magnetic part. The most accurate experimental values of the related transitions are

$$
\begin{aligned}
\nu(1 s-2 s) & =2455528941.0(9.8) \mathrm{MHz}, \quad[1] \\
\nu(1 s, \mathrm{hfs}) & =4463302.776(51) \mathrm{kHz}, \quad[2] .
\end{aligned}
$$

Comparison of theory and experiments provides one of the most accurate tests of quantum electrodynamics (QED) calculations (see, 3, 4] for details).

Since it is possible to control relativistic, recoil and QED corrections, one can apply these data to check whether a leading non-relativistic term obtained from the one-photon exchange is consistent with the gross experimental picture and to constrain possible deviations from the standard description.

The leading terms are

$$
\begin{aligned}
\nu(1 s-2 s) & \simeq \frac{3}{4} R_{\infty}, \\
\nu(1 s, \mathrm{hfs}) & \simeq E_{\mathrm{F}}=\frac{16 \alpha}{3 \pi} \mu_{\mathrm{B}} \mu_{\mu} R_{\infty} m_{e}^{2},
\end{aligned}
$$

where we apply relativistic units in which $\hbar=c=1$, $e^{2} /(4 \pi)=\alpha$ is the fine structure constant, $m_{e}$ is the electron mass and $R_{\infty}$ is the Rydberg constant, $\mu_{\mathrm{B}}=$ $e /\left(2 m_{e}\right)$ is the Bohr magneton and $\mu_{\mu}$ is the muon magnetic moment. Similar equations hold for related transitions in other light hydrogen-like atoms.

To calculate the dominant contributions, one has at first to determine the values of related fundamental constants, such as the fine structure constant $\alpha$, the Rydberg

*Electronic address: savely.karshenboim@mpq.mpg.de constant $R_{\infty}$, the muon magnetic moment $\mu_{\mu}$. The latter come from a bunch of experiments involved in the data analysis [5] and if a certain 'new-physics' effect would affect those experiments in a different way, the outcome could be inconsistent.

In this paper we study a possible inconsistency in the interpretation of different experiments caused by an exchange by a pseudovector boson with a light mass and an ultraweak coupling.

Such a boson should affect the HFS interval, which has been measured with high accuracy in a number of light two-body atoms.

The strongest constraint we derive here is from muonium physics, on which we focus our attention and consider related data in details. Weaker constraints from experiments on other atoms are also considered.

The constraint on the exchange by an intermediate boson comes from its one-particle-exchange (OPE) contribution. Meantime, even in the OPE approximation there are other intermediate particles which contribute to the effective muon-electron interaction and shift the energy levels.

Some of such small contributions have been already included into consideration of the muonium HFS as corrections due to weak and strong interactions. At the tree level a $Z$ boson exchange should be included (see, e.g., [6]). When taking into account various perturbative effects and corrections one has to consider exchange by $\rho$ meson, pion, $a_{1}$-meson etc. Those hadronic OPE contributions are a result of certain reduction of more complicated graphs to tree level. In particular, the $\rho$ exchange is taken into account when considering the hadronic vacuum polarization contribution [7], while $\pi^{0}$ and $a_{1}$ exchange is a part of consideration of the hadronic lightby-light-scattering contribution [8].

In contrast to the situation with the anomalous magnetic moment of a muon (see, e.g., [9]), the mentioned corrections $\left(Z, \rho, \pi^{0}, a_{1}\right)$ to the one-photon exchange are very small and are of marginal importance for the present level of experimental and theoretical accuracy in spectroscopy of muonium and other simple atoms. Their smallness comes from the large masses of the intermedi- 
ate particles (all are much heavier than electron), while in the hadronic case each induced vertex is additionally suppressed, because it involves higher-order electrodynamics effects and thus involves extra factors of $\alpha$.

Various unification theories (see, e.g. [10, 11]) may involve a particle lighter than an electron and here we constrain its coupling constant.

A constraint on a possible new spin-independent interaction from precision physics of simple atoms has been already considered [12, 13] and here we discuss a spin-dependent interaction, which is somewhat similar to those due to exchange by $Z, \rho, \pi^{0}, a_{1}$. The characteristic momentum at the ground state is about $4 \mathrm{keV}$ and we consider an intermediate particle lighter than that.

Two of mentioned interactions, namely due to $Z$ and $a_{1}$ exchange, are of our particular interest, because they produce a spin-spin coupling directly. By introducing such a spin-spin interaction, the Coulomb exchange should be corrected

$$
-\frac{\alpha}{r} \rightarrow-\frac{\alpha+\alpha^{\prime \prime}\left(\mathbf{s}_{e} \cdot \mathbf{s}_{\mu}\right) \mathrm{e}^{-\lambda r}}{r} .
$$

Such a correction is specific for a pseudovector particles. For instance, vector (e.g. a photon) or pseudoscalar (e.g. an axion) particles produces a spin-spin interaction only as a relativistic effect. In particular, in the case of spin $1 / 2$ as long as the large components of the Dirac wave function are considered the spin-involving effects do not appear. The vector-particle exchange is a kind of static electric interaction and the axion-induced interaction is vanishing. Once we include the small components the vector-particle exchange involve the magnetic forces and the axion exchange becomes observable. Including of small components of both interacting particles produces a suppression factor of $1 /\left(m_{1} m_{2} r^{2}\right) \ll 1$, which dramatically weaken the spin-dependent constraints on pseudoscalar particles (while for vector particles more strong constraints originates indeed from spin-independent interaction (see, e.g., [13]).

Studying the spin-spin interaction we can simplify (3) under certain conditions. In particular, if the intermediate particle is massless (or, which is the same, lighter than $4 \mathrm{keV}$ ), the resulting interaction at atomic scale should be

$$
-\frac{\alpha}{r} \rightarrow-\frac{\alpha+\alpha^{\prime \prime}\left(\mathbf{s}_{e} \cdot \mathbf{s}_{\bar{\mu}}\right)}{r} .
$$

In principle, such a mechanism, involving a new particle, can produce some ultraweak spin-dependent longrange interaction.

Here we consider an interaction, which is similar to $Z$ and $a_{1}$ exchange, but with different strength and mass. A possible range of $\alpha^{\prime \prime}$ for the mass of the intermediate particle (i.e., of the radius of the interaction) below $4 \mathrm{keV}$ is under our investigation [12].

Such an intermediate particle is coupled to charged particles and is rather expected to be unstable and to decay into photons. However, as long as its width is much smaller than its mass, we can consider the particle as stable while calculating the related corrections to the energy levels (cf. with calculations of the $Z$ [6] and $a_{1}$ [8] exchange for the muonium HFS).

\section{METHOD}

The HFS interval in light hydrogen-like atoms can be expressed in terms of the so-called Fermi energy $E_{\mathrm{F}}$ and a correcting factor due to reduced-mass, relativistic, recoil and QED effects. Taking the latter into account [3, 4] one can interpret any measurement of the actual HFS interval as a measurement of $E_{\mathrm{F}}$. The Fermi energy (see, e.g. (2) for the muonium Fermi energy) is in its turn proportional to a product of the muon and electron magnetic moments.

The ground state HFS has been studied with a high accuracy in six two-body atoms, which are muonium [2], positronium [14], hydrogen [15], deuterium [16], tritium 17] and helium-3 ion [18]. The strength of the constraint on a light pseudovector meson depends not only on accuracy of the experimental determination of the HFS interval, but also on accuracy of the theoretical calculation of this quantity. We briefly overview the related theoretical problems in Sect. V] Below, we focus our attention on muonium, study of which delivers us the strongest constraint on $\alpha^{\prime \prime}$.

At present, a way of an $E_{\mathrm{F}}$ calculation is the following: one takes an experimental value of $\mu_{\mu}$ and uses it in the calculation. The value is obtained in macroscopic measurements, say at $r>1 \mathrm{~cm}$. If the interaction we are to constrain is related to the case

$$
1 \mathrm{~cm}^{-1} \ll \lambda \ll 4 \mathrm{keV},
$$

then a certain mismatch, proportional to $\alpha^{\prime \prime}$ should appear because the spin-spin term in (4) also contributes to the HFS interval. The HFS interval is shifted by

$$
\delta E_{\mathrm{hfs}}=-\frac{Z^{2}\left(\alpha+\alpha^{\prime \prime}\right)^{2} m_{r}}{2}+\frac{Z^{2} \alpha^{2} m_{r}}{2} .
$$

This correction is universal for atoms with the nuclear spin $1 / 2$. (For the nuclear spin 1, e.g., in deuterium, a factor of $3 / 2$ should be introduced.) Here, $m_{r}$ is the reduced mass, which for all atoms under study but positronium, is equal to the electron mass $m_{e}$ with a sufficient accuracy. In positronium, indeed, $m_{r}=m_{e} / 2$.

The correction can be rewritten in terms of an effective correction to magnetic moment in such a way that the Fermi energy with a 'corrected' magnetic moment includes a correction (6).

In the case of muonium it is of the form

$$
\delta E_{\mathrm{hfs}}=\frac{16 \alpha}{3 \pi} \mu_{\mathrm{B}} \mu_{\mu}^{\prime} R_{\infty} m_{e}^{2},
$$


where $\mu_{\mu}^{\prime}$ is defined as

$$
\begin{aligned}
\mu_{\mu}^{\prime} & =-\mu_{\mu} \times \frac{2 \alpha^{\prime \prime}}{\alpha} \frac{R_{\infty}}{E_{\mathrm{F}}} \\
& =-2.0 \times 10^{8} \mu_{\mu} \alpha^{\prime \prime} .
\end{aligned}
$$

That is the value of $\mu_{\mu}^{\prime}$ that should appear as a mismatch in a determination of the muon magnetic moment, found from a comparison of Eqs. (2) and (11) and taking into account all necessary reduced-mass, relativistic, recoil and QED corrections [3 [5], and a value, obtained by a 'direct' macroscopic measurement. Determination of magnetic moments of various particles (muon, proton) and light nuclei (deuteron, triton, helion (the nucleus of the helium-3)) is reviewed in detail in [5] (see Sect. VI there).

\section{DETERMINATION OF THE MUON MAGNETIC MOMENT}

Let us consider a determination of the muon magnetic moment. The most accurate value (in units of the proton magnetic moment) is [5]

$$
\frac{\mu_{\mu}}{\mu_{p}}=3.183345137(85) .
$$

The result is obtained after evaluation of all the world data. The dominant contribution comes from a comparison of (2) and (11) with all appropriate corrections taken, while the other measurements are statistically negligible. That is not a value obtained by any 'direct' means.

If the correction (8) is present, we should interpret this result as

$$
\frac{\mu_{\mu}+\mu_{\mu}^{\prime}}{\mu_{p}}=3.183345137(85) .
$$

This value should be compared with a 'direct' measurement [2]

$$
\frac{\mu_{\mu}}{\mu_{p}}=3.18334524(37) .
$$

The latter is derived from a study of Breit-Rabi magnetic sublevels of the ground state in the magnetic field. So, it is determined from a macroscopic experiment.

The discussion on $\mu_{\mu}$ above involves also $\mu_{p}$ (see Eqs. (10 and (11)) as a unit. It appears in $E_{F}$, where the actual dimensionless factor reads $\mu_{\mu} m_{e} / e=$ $\left(\mu_{\mu} / \mu_{p}\right)\left(\mu_{p} / \mu_{\mathrm{B}}\right)\left(\mu_{\mathrm{B}} m_{e} / e\right)$. Note that $\mu_{\mathrm{B}} m_{e} / e=1 / 2$, while the factor $\mu_{p} / \mu_{\mathrm{B}}$ is determined from macroscopic experiments. Different scales are related only to two determinations of $\mu_{\mu}$ and do not touch any other involved quantities. The value of $\mu_{p}$ is customarily involved in a presentation of the results but does not play any real role in the issue under consideration.

For the references on measurements of these and similar quantities, useful to examine HFS intervals in other light atoms, let us mention that the magnetic moments of proton [19] and deuteron [20] in units of the electron magnetic moment are determined from a study of similar level structure as in muonium [2], while the magnetic moments of triton 21, 22] and helion 23] are obtained from NMR spectroscopy (see also [21] for an NMR determination of $\mu_{p} / \mu_{d}$ ). To convert a result obtained in terms of $\mu_{e}$ into results in terms of $\mu_{\mathrm{B}}$, one has to apply a value of $g_{e}$ measured in a macroscopic experiment [24] as well.

\section{CONSTRAINING A LONG-RANGE SPIN-SPIN INTERACTION FROM MUONIUM HFS}

The constraint on the electron-antimuon spindependent coupling constant, resulting from comparison of (10) and (11), reads

$$
\alpha^{\prime \prime}=(1.6 \pm 6.0) \times 10^{-16},
$$

which is the major result of the paper.

As already mentioned, the 'direct' measurement deals with behavior of hyperfine energy levels in macroscopic magnetic field [2]. A value of $\mu_{e} / \mu_{p}$ has also been used as an input datum that was obtained from measurements of splitting of Breit-Rabi HFS sublevels in magnetic field [19] (for an adequate theory see [4, 5, 28]) at the macsoscopic distance scale.

To be conservative, we estimate the distances from the field source at the mentioned macroscopic experiments as larger than $1 \mathrm{~cm}$. In principle, one could consider a comparison of the atomic scale with a somewhat larger distance. However, in this case it is necessary to completely reanalyze both quoted experiments for their magnetic effects, including the source of the field and the shielding applied. In any case, from the point of view of particle physics that is rather a higher-energy end for the scale which is of interest. That range is related to the mass of an intermediate boson of roughly $4 \mathrm{keV}$.

\section{CONSTRAINTS FROM THE GROUND STATE HFS INTERVAL IN OTHER LIGHT H-LIKE ATOMS}

The constraint (12) is related to a four-fermion $e \bar{e}-\mu \bar{\mu}$ interaction. Some other interactions can be also constrained from atomic physics. A similar constraint can be also set for an $e \bar{e}-e \bar{e}$ interaction, but it is weaker by a few orders of magnitude. That is because positronium HFS is much larger (i.e., the HFS is a bigger portion of the Rydberg energy) and because of lower experimental accuracy in the determination of the HFS interval [14]. The constraint is at the level of a few parts in $10^{12}$. Since theory and experiment disagree at the level of about $2.5 \sigma$ (see, e.g., [4]), perhaps, we have to estimate the experimental and theoretical uncertainty somewhat more con- 
servatively than in the original publications. In any case, in such a specific area as a constraint on 'new physics', the aim is rather a conservative limitation than a 'detection', and some two- or three-sigma effects are observed from time to time.

In the case of an $e \bar{e}-p \bar{p}$ interaction, accuracy should be also somewhat lower than (12) because of relatively low theoretical accuracy, the uncertainty of which is due to the proton structure effects (see, e.g., [3, 4] ). That is compensated in part by a smaller value of the HFS interval because of smaller value of the nuclear magnetic moment (cf. (8) ).

A constraint for a compound particle can be derived, e.g., from the deuteron HFS. The theoretical accuracy here is even worse than for hydrogen (see, e.g., [4]), but the enhancement because of a small HFS interval is larger.

The exact value of constraints for $\alpha^{\prime \prime}$ for an $e \bar{e}-p \bar{p}$ and $e \bar{e}-d \bar{d}$ interaction should come from an estimation of the nuclear-structure-uncertainty. It seems, however, that a situation with that for the HFS intervals in hydrogen and deuterium is somewhat uncertain and we prefer to give a rough estimation. We expect a constraint on the related $\alpha^{\prime \prime}$ values at the level of a few parts in $10^{15}$. Utilization of data from other light atoms is also possible.

A study of the HFS structure of other light atoms, such as tritium and helium-3 ion, can provide similar constraints after the contribution and uncertainty of their nuclear-structure effects are properly estimated.

The results are summarized in Table I. We emphasize that the constraints for non-leptonic atoms are rough estimations and the accuracy of understanding of the nuclear structure effects requires clarification.

\begin{tabular}{cr}
\hline Atom & \multicolumn{1}{c}{$\alpha^{\prime \prime}$} \\
\hline $\mathrm{Mu}$ & $(1.6 \pm 6.0) \times 10^{-16}$ \\
$\mathrm{Ps}$ & $(5.8 \pm 2.1) \times 10^{-12}$ \\
$\mathrm{H}$ & $\pm 1.6 \times 10^{-15}$ \\
$\mathrm{D}$ & $\pm 8 \times 10^{-15}$ \\
$\mathrm{~T}$ & $\pm 7 \times 10^{-14}$ \\
${ }^{3} \mathrm{He}^{+}$ & $\pm 5 \times 10^{-13}$ \\
\hline
\end{tabular}

TABLE I: The constraint from the $1 s$ HFS intervals on a coupling constant $\alpha^{\prime \prime}$ for a pseudovector boson with mass $\lambda \ll \alpha m_{e} \simeq 3.5 \mathrm{keV}$ (which is related to the Yukawa radius substantially above $\left.a_{0}\right)$.

All constraints, but the one from positronium, are based on a comparison of a certain HFS interval and the related nuclear magnetic moment, determined at macroscopic distances [2, 5, 19 23]. For a specific case of positronium, the leading term for the HFS interval

$$
E_{F}(\mathrm{Ps})=\frac{7}{6} \alpha^{2} R_{\infty}
$$

is calculated only from the knowledge of electron charge and mass, which enter in combinations, determination of which is insensitive to any spin-dependent interaction.
(One has to remember that a determination of the fine structure constant can be done by many methods and some of them do not involve any magnetic effects. In particular, one can find $\alpha$ from $R_{\infty}$ and a certain $h / M$ value [25, 26] as discussed in [5] and [13].) That allows to extend the constraint to larger distances.

More detail on the data used for constraining the spindependent long-range interaction from the $1 s$ HFS can be found in Appendix A

\section{EXTENDING THE CONSTRAINTS TO A LARGER-MASS RANGE}

A constraint from the value of the $1 s$ HFS interval can be easily extended to higher values of the mass of the intermediate boson, $\lambda$. A direct calculation of the contribution of the Yukawa spin-dependent term in (3) leads to

$$
\delta E_{\mathrm{hfs}}=-2 \frac{\alpha^{\prime \prime}}{\alpha^{2}} Z^{2} \frac{m_{r}}{m_{e}} R_{\infty} \times \mathcal{F}_{1}\left(\lambda / Z \alpha m_{r}\right),
$$

and so

$$
\alpha^{\prime \prime}(\lambda)=\frac{\alpha_{0}^{\prime \prime}}{\mathcal{F}_{1}\left(\lambda / Z \alpha m_{r}\right)} .
$$

Here $\alpha_{0}^{\prime \prime}$ is a related constraint for $\lambda \ll Z \alpha m_{e}=$ $3.5 Z \mathrm{keV}$, listed in Table I, and the profile function is of the form

$$
\mathcal{F}_{1}(x)=\left(\frac{2}{2+x}\right)^{2} .
$$

The constraint for the extended $\lambda$ range is presented in Fig. 11 [12]. Here, the nuclear charge $Z$ is unity for all atoms, but helium-3 ion $\left(Z_{h}=2\right)$ and the reduced mass $m_{r}$ is equal to the electron mass $m_{e}$ for all atoms, but positronium $\left(m_{r}(\mathrm{Ps})=m_{e} / 2\right)$. Because of that, the mass dependence of positronium and helium constraints is somewhat different from results derived from muonium, hydrogen, deuterium and tritium.

\section{SUMMARY}

The final constraints from the $1 s$ HFS in six different two-body atoms are summarized in Fig. 11 12]. For the atomic systems, where the nuclear-effects are not well estimated, we apply rather a rough estimation which, in principle, can be substantially improved. The strength of constraint for $\alpha_{0}^{\prime \prime}$ from muonium and positronium is determined by experimental accuracy. For muonium that is the experimental accuracy of determination of the muon magnetic moment in appropriate units. For positronium the dominant uncertainty is the one for measurements of the $1 s$ HFS interval, while the theoretical uncertainty is smaller, but still comparable with the experimental one. 


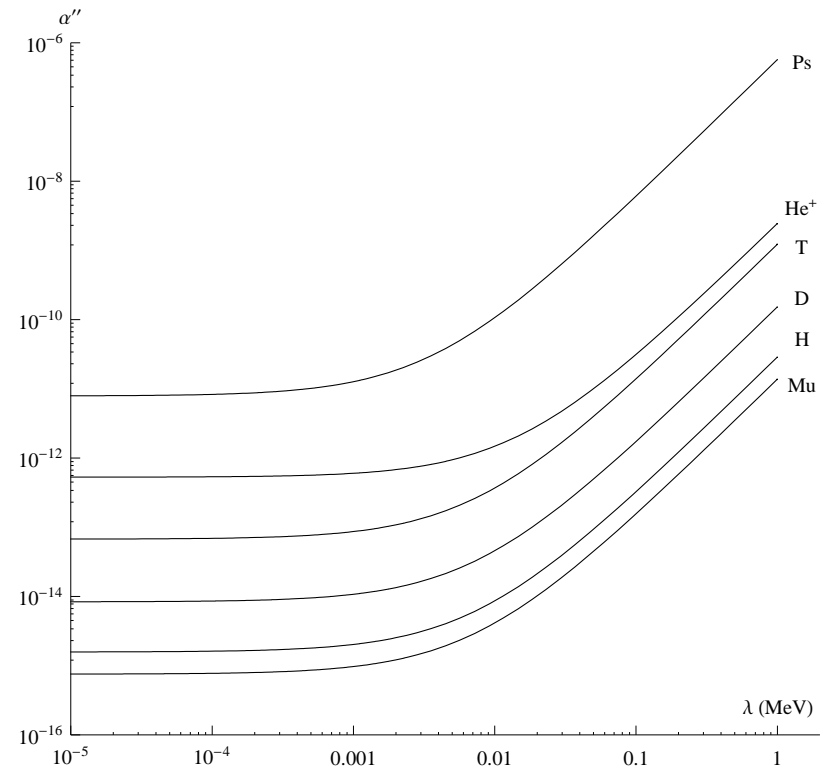

FIG. 1: Constraint on a long-term spin-dependent interaction from the HFS intervals of the ground state in light two-body atoms. The lines present the upper bound on $\left|\alpha^{\prime \prime}\right|$ from data on the $1 s$ HFS interval in muonium, hydrogen, deuterium, tritium, helium-3 ion, and positronium. A mass of an intermediate particle $\lambda$ is the inverse Yukawa radius. The confidence level is related to one standard deviation.

For hydrogen [15], deuterium [16], tritium [17] and helium-3 ion [18] the experimental data are substantially more accurate, and the strength is determined by an uncertainty in understanding the nuclear effects, which limits the theoretical accuracy (see, e.g., [4]).

One can note that the spin-dependent constraint obtained here is stronger comparing with our spinindependent constraints [13], which, in fact, deal with substantially more accurate data. A reason for that is that the interaction (4) modifies the Coulomb interaction and thus an enhancement factor (i.e., a factor of $2 \times 10^{8}$ in (8) ) comparing with the magnetic interaction appeared.

It is also notable that the constrained boson is responsible for a kind of interaction, which is somewhat similar to the weak interaction by $Z$ boson exchange. While the weak contribution to the $1 s$ HFS in light hydrogenlike atoms is below the accuracy of comparison of theory and experiment (see, e.g. [3-5]), our constraint is quite strong.

Concerning the weak interaction in atomic physics, we have to remind that the weak interaction is weaker than the electromagnetic one not because of a weak coupling constant, but because of the heaviness of its intermediate particle. Introducing a new particle, the correction would increase with a lighter mass and decrease with a weaker coupling constant. From the point of view of the final result, a correction with near-zero mass and an ultraweak interaction can be compatible with a conven- tional weak interaction. Indeed, that is possible only at the low momentum transfer. In similar matter various atomic weak-interaction experiments can also constrain certain long-range interaction.

Concluding, here, we constrain not just a pseudovector particle, but a particle with spin one and axial coupling. That covers not only pseudo vectors, but also a light particle, which similarly to $Z$ boson, does not have a fixed parity. The weak interaction experiments in atomic physics are unlikely useful to constrain light pseudovectors, however, it seems that constraining the pseudo vector we also constraint corrections to the weak interaction at atomic distances.

\section{Acknowledgements}

This work was supported in part by RFBR (grants \#\# 08-02-91969 \& 08-02-13516) and DFG (grant GZ 436 RUS $113 / 769 / 0-3)$. The author is grateful to Andrej Afanasev, Dmitry Toporkov, Eugene Korzinin, Simon Eidelman, Maxim Pospelov, and Oleg Sushkov for useful and stimulating discussions.

\section{Appendix A: Summary on experimental and theoretical data on the $1 s$ HFS interval in light two-body atoms}

Here, we collect reference data applied in the evaluation. The experimental results on the $1 s$ HFS interval are collected in Table III They represent physics at atomic scale.

\begin{tabular}{ccc}
\hline Atom & $\begin{array}{c}E_{\mathrm{HFS}}(\exp ) \\
{[\mathrm{kHz}]}\end{array}$ & Refs. \\
\hline Muonium & $4463302.78(5)$ & {$[2]$} \\
Hydrogen & $1420405.751768(1)$ & {$[15]$} \\
Deuterium & $327384.352522(2)$ & {$[16]$} \\
Tritium & $1516701.470773(8)$ & {$[17]$} \\
${ }^{3} \mathrm{He}^{+}$ion & $-8665649.867(10)$ & {$[18]$} \\
Positronium & $203389100(740)$ & {$[14]$} \\
\hline
\end{tabular}

TABLE II: The most accurate results for the $1 s$ HFS interval in light hydrogen-like atoms. A negative sign for the ${ }^{3} \mathrm{He}^{+}$ion reflects the fact that the nuclear magnetic moment is negative, i.e., in contrast to other nuclei in the Table, its direction is antiparallel to the nuclear spin.

To constrain a long-range interaction, one has to compare it with physics on macroscopic distances, which provides a value of the magnetic moment of the involved nuclei. We summarize in Table III results on $\mu_{\text {nucl }} / \mu_{B}$, values of magnetic moments of the nuclei of interest in units of the Bohr magneton.

Determination of the nuclear magnetic moment for light atoms is mostly done for bound nuclei. A number of measurements are done on the Breit-Rabi levels in 


\begin{tabular}{llll}
\hline Quantity & $\begin{array}{c}\text { Value } \\
{\left[\times 10^{-3}\right]}\end{array}$ & \multicolumn{1}{c}{ Method } & Refs. \\
\hline$\mu_{\mu} / \mu_{\mathrm{B}}$ & $4.84197049(12)$ & BR Mu @ B \& NMR p $[2]$ \\
$\mu_{p} / \mu_{\mathrm{B}}$ & $1.521032209(12)$ & BR H @ B & {$[19]$} \\
$\mu_{d} / \mu_{\mathrm{B}}$ & $1.04187563(25)$ & BR D @ $B$ & {$[20]$} \\
& & and BR H \& NMR HD $[21]$ \\
$\mu_{t} / \mu_{\mathrm{B}}$ & $1.622393657(21)$ & NMR HT & {$[21,22]$} \\
$\mu_{h} / \mu_{\mathrm{B}}$ & $1.158741958(14)$ & NMR He \& $\mathrm{H}_{2} \mathrm{O}$ & {$[23,27]$} \\
\hline
\end{tabular}

TABLE III: Determination of the nuclear magnetic moment in light atoms. 'BR @ $B$ ' is for study of the Breit-Rabi levels at presence of magnetic field (in muonium, hydrogen and deuterium); 'NMR' stands for nuclear magnetic resonance of free protons (p), atoms of ${ }^{3} \mathrm{He}$ and molecules of $\mathrm{HD}$, HT and $\mathrm{H}_{2} \mathrm{O}$. The references and description of the method are given for the most crucial measurements only. ' $h$ ' stands for helion, the nucleus of the helium-3 atom. All results, but result for the helion, the nucleus of ${ }^{3} \mathrm{He}$, are taken directly from [5] . The helion result is obtained as explained in the text.

two-body atoms and the shielding correction is discussed in [5, 28]. The most complicated is theory of the neutral helium-3 atom, a three-body system, where theory with sufficient accuracy is presented in [29]. Other experiments are performed with the nuclear magnetic resonance technique on diatomic molecules HD and HT. They are more complicated for calculations, however, it is the ratio of nuclear magnetic moments (deuterium-to-proton and tritium-to-proton) that is measured and this kind of isotopic calculations has relatively high accuracy (see 21] for detail).

All the references and description of the method are given in the table for the most crucial measurements for each involved nucleus. Other involved measurements were on the anomalous magnetic moments of electron and muon and on the proton-to-electron mass ratio. All three values are known with accuracy much better than required (see [5] for detail). All results, but a result for the helion, the nucleus of ${ }^{3} \mathrm{He}$, are taken from the CODATA tables of recommended values [5]. To obtain the free helion value we used the related shielded value of $1.158671,471(14) \times 10^{-3}$ from [5] and the shielding factor $\left(\sigma=59.96743(10) \times 10^{-6}\right)$ recently calculated in $[29]$.

\begin{tabular}{|c|c|c|}
\hline Atom & $\begin{array}{c}\text { Fractional } \\
\text { uncertainty }\end{array}$ & $\begin{array}{c}\text { Dominant source } \\
\text { of uncertainty }\end{array}$ \\
\hline Muonium & $0.12 \mathrm{ppm}$ & determination of $\mu_{\mu} / \mu_{p}$ \\
\hline Hydrogen & $1 \mathrm{ppm}$ & nuclear effects \\
\hline Deuterium & $35 \mathrm{ppm}$ & nuclear effects \\
\hline Tritium & $40 \mathrm{ppm}$ & nuclear effects \\
\hline${ }^{3} \mathrm{He}^{+}$ion & 200 ppm & nuclear effects \\
\hline Positronium & $4.4 \mathrm{ppm}$ & experiment \& theory \\
\hline
\end{tabular}

TABLE IV: Uncertainty of comparison of theory and experiment for the $1 s$ HFS interval in light hydrogen-like atoms.

To conclude a short overview of involved values and accuracies we summarize in Table IV the uncertainty of comparison of the experiment and theory of the $1 s$ HFS interval in light two-body atoms. While the accuracy for muonium and positronium is well understood and was numerously discussed in literature (see, e.g., [4]), the uncertainty for conventional atoms in the table is rather a rough estimation accepted in this paper.
[1] V. Meyer, S. N. Bagayev, P. E. G. Baird, P. Bakule, M. G. Boshier, A. Breitrück, S. L. Cornish, S. Dychkov, G. H. Eaton, A. Grossmann, D. Hübl, V. W. Hughes, K. Jungmann, I. C. Lane, Yi-Wei Liu, D. Lucas, Y. Matyugin, J. Merkel, G. zu Putlitz, I. Reinhard, P. G. H. Sandars, R. Santra, P. V. Schmidt, C. A. Scott, W. T. Toner, M. Towrie, K. Träger, L. Willmann, and V. Yakhontov, Phys. Rev. Lett. 84, 1136 (2000).

[2] W. Liu, M. G. Boshier, S. Dhawan, O. van Dyck, P. Egan, X. Fei, M. G. Perdekamp, V. W. Hughes, M. Janousch, K. Jungmann, D. Kawall, F. G. Mariam, C. Pillai, R. Prigl, G. zu Putlitz, I. Reinhard, W. Schwarz, P. A. Thompson, and K. A. Woodle, Phys. Rev. Lett. 82, 711 (1999).

[3] M.I. Eides, H. Grotch and V.A. Shelyuto, Phys. Rep. 342, 63 (2001);

M.I. Eides, H. Grotch and V.A. Shelyuto, Theory of Light Hydrogenic Bound States, Springer Tracts Mod. Phys. 222 (Springer, Berlin, Heidelberg, 2007).

[4] S. G. Karshenboim, Phys. Rep. 422, 1 (2005).

[5] P. J. Mohr, B. N. Taylor, and D. B. Newell, Rev. Mod. Phys. 80, 633 (2008).

[6] M. I. Eides, Phys. Rev. A53, 2953 (1996).

[7] J. R. Sapirstein, E. A. Terray, and D. R. Yennie, Phys. Rev. D29, 2290 (1984);
R.N. Faustov, A. Karimkhodzhaev and A.P. Martynenko, Phys. Rev. A59, 2498 (1999);

A. Czarnecki, S. I. Eidelman and S. G. Karshenboim, Phys. Rev. D65 (2002);

S.I. Eidelman, S.G. Karshenboim and V.A. Shelyuto, Can. J. Phys. 80, 1297 (2002).

[8] S.G. Karshenboim, V.A. Shelyuto and A.I. Vainshtein, Phys. Rev. D78, 065036 (2008).

[9] K. Melnikov and A. Vainshtein, Theory of the Muon Anomalous Magnetic Moment. Springer Tracts Mod. Phys. 216 (Springer, Berlin, Heidelberg, 2006);

F. Jegerlehner, The Anomalous Magnetic Moment of the Muon. Springer Tracts Mod. Phys. 222 (Springer, Berlin, Heidelberg, 2008);

F. Jegerlehner and A. Nyffeler, Phys. Rep. 477, 1 (2009).

[10] B. Holdom, Phys. Lett. 166B, 196 (1986).

[11] M. Pospelov, Phys. Rev. D80, 095002 (2009).

[12] S.G. Karshenboim, Phys. Rev. Lett. (2010) to be published; eprint arXiv:1005.4859.

[13] S.G. Karshenboim, eprint arXiv:1005.4872.

[14] M. W. Ritter, P. O. Egan, V. W. Hughes and K. A. Woodle, Phys. Rev. A30, 1331 (1984);

A. P. Mills, Jr., and G. H. Bearman, Phys. Rev. Lett. 34, 246 (1975); 
A. P. Mills, Jr., Phys. Rev. A27, 262 (1983).

[15] H. Hellwig, R.F.C. Vessot, M. W. Levine, P. W. Zitzewitz, D. W. Allan, and D. J. Glaze, IEEE Trans. IM-19, 200 (1970);

P. W. Zitzewitz, E. E. Uzgiris, and N. F. Ramsey, Rev. Sci. Instr. 41, 81 (1970);

L. Essen, R. W. Donaldson, E.G. Hope and M. J. Bangham, Metrologia 9, 128 (1973);

D. Morris, Metrologia 7, 162 (1971);

V. S. Reinhard and J. Lavanceau, in Proceedings of the 28th Annual Symposium on Frequency Control (Fort Mammouth, N. J., 1974), p. 379;

P. Petit, M. Desaintfuscien and C. Audoin, Metrologia 16, 7 (1980);

J. Vanier and R. Larouche, Metrologia 14, 31 (1976);

Y. M. Cheng, Y. L. Hua, C. B. Chen, J. H. Gao and W. Shen, IEEE Trans. IM-29, 316 (1980);

S. G. Karshenboim, Can. J. Phys. 78, 639 (2000).

[16] D. J. Wineland and N. F. Ramsey, Phys. Rev. 5, 821 (1972).

[17] B. S. Mathur, S. B. Crampton, D. Kleppner and N. F. Ramsey, Phys. Rev. 158, 14 (1967).

[18] H. A. Schluessler, E. N. Forton and H. G. Dehmelt, Phys. Rev. 187, 5 (1969).

[19] P. F. Winkler, D. Kleppner, T. Myint and F. G. Walther, Phys. Rev. A 5, 83 (1972); corrected according to a private communication with $\mathrm{D}$. Kleppner as quoted in [5].
[20] W. D. Phillips, D. Kleppner, and F. G. Walther, private communication; quoted according to [5].

[21] Yu. I. Neronov and S. G. Karshenboim, Phys. Lett. A318, 126 (2003).

[22] Yu. I. Neronov and A. E. Barzakh, Sov. Phys. JETP 45, 871 (1977).

[23] J. L. Flowers, B. W. Petley, and M. G. Richards, Metrologia 30, 75 (1993).

[24] D. Hanneke, S. Fogwell, and G. Gabrielse, Phys. Rev. Lett. 100, 120801 (2008)

[25] A. Wicht, J. M. Hensley, E. Sarajlic, and S. Chu, Phys. Scr. T102, 82 (2002);

A. Wicht, E. Sarajlic, J. M. Hensley, and S. Chu, Phys. Rev. A72, 023602 (2005).

[26] M. Cadoret, E. de Mirandes, P. Cladé, S. GuellatiKhélifa, C. Schwob, F. Nez, L. Julien, and F. Biraben, Phys. Rev. Lett. 101, 230801 (2008).

[27] W. D. Phillips, W. E. Cooke, and D. Kleppner, Metrologia 13, 179 (1977);

B. W. Petley and R. W. Donaldson, Metrologia 20, 81 (1984).

[28] S. G. Karshenboim and V. G. Ivanov, Phys. Lett. B 566, 27 (2003).

[29] A. Rudzinski, M. Puchalski, and K. Pachucki, J. Chem. Phys. 130, 244102 (2009). 